\title{
Múltipla Deficiência e Baixa Visão
}

\author{
Maria Inês R.S. Nobre* \\ Heloisa G.R.G. Gagliardo** \\ K. Monteiro de Carvalho*** \\ Marilda B.S. Botega ${ }^{\star \star * *}$ \\ Paulo R. Sampaio ${ }^{\star \star \star \star *}$
}

\begin{abstract}
RESUMO
Crianças com múltipla deficiência apresentam com freqüência deficiência visual associada. Geralmente, as crianças com quadro neurológico grave têm o quadro motor intensamente comprometido, sendo esse priorizado em programas de habilitação infantil. Devido à dificuldade em se observar as respostas visuais nessas criancas, normalmente o possível diagnóstico oftalmológico é tardio, comprometendo ainda mais seu desenvolvimento.

Durante um período de 3 meses, em um serviço de visão subnormal, os autores encontraram uma freqüência de $50 \%$ de crianças com múltipla deficiência associada à deficiência visual. Verificou-se que a média de chegada dessas crianças ao serviço foi de 21 meses, sendo todas institucionalizadas.

Conclui-se que o comprometimento neuromotor pode retardar o encaminhamento dessas crianças ao oftalmologista. As que apresentam resíduo visual podem ser privadas de estimulação adequada nos períodos sensíveis de desenvolvimento da visão, podendo ter prejudicado seu desenvolvimento visual e, em conseqüência, o desenvolvimento neuromotor.
\end{abstract}

\section{UNITERMOS}

Visão subnormal, desenvolvimento neuromotor, múltipla deficiência.
Mestre em Neurociências. Terapeuta Ocupacional - CEPRE-FCM-UNICAMP.

** Mestre em Neurociências. Terapeuta Ocupacional - CEPRE-FCM-UNICAMP.

*** Profa Dra. Médica Oftalmologista. Departamento de Oftalmologia - FCM-UNICAMP.

**** Fonoaudióloga - CEPRE/FCM-UNICAMP.

***** Médico Oftalmologista - Departamento de Oftalmologia - FCM-UNICAMP.

\section{INTRODUÇÃO}

O nível de gravidade dos comprometimentos em crianças multideficientes tem aumentado nos últimos anos, principalmente pelo alto índice de prematuros que sobrevivem graças ao progresso da medicina pré-natal e neonatal. O potencial dessas crianças era, em muitos casos, subestimado, ora pelas reais dificuldades e pela não-clareza dos diagnósticos, ora pela escassez de terapeutas especializados nessa área. Hoje, muitos desses problemas foram superados, mas alguns ainda persistem.

Segundo a nomenclatura do Centro Técnico Nacional Francês, para a identificação desse grupo de crianças "diferentes", muitos termos têm sido utilizados: crianças com deficiências associadas, multideficientes, plurideficientes, multincapacitados.

Zaldivar \& Rubio $^{1}$ definem como pessoas plurideficientes aquelas que apresentam simultaneamente uma combinação de deficiências sensoriais, psíquicas e/ou físicas. Para esses autores, falar de pessoas plurideficientes implica algo mais que falar em uma ou mais deficiências. Os plurideficientes são indivíduos com uma combinação particular de déficits, que se interrelacionam, estabelecendo determinadas limitações em cada pessoa.

Há uma grande dificuldade em se definir essas crianças. Segundo a Organização Mundial da Saúde (OMS), a incapacidade é definida como toda restrição ou ausência da capacidade de realizar uma atividade na forma ou dentro da margem que se considera normal para o ser humano.

Partimos do princípio de que crianças com múltipla deficiência apresentam uma ampla gama de necessidades e, dificilmente, uma única pessoa estará apta a solucionar todas as necessidades dessas crianças. Sendo assim, elas devem ser atendidas por uma equipe multidisciplinar.

Devido à complexidade na evolução dessas crianças, sugerimos avaliações em intervalos pequenos.

A criança com múltipla deficiência, em geral, chega tardiamente ao serviço de estimulação visual; isso pode ser devido à gravidade neurológica dos casos, 
dificuldade de acesso aos centros especializados, dificuldade para se definir o diagnóstico ou, ainda, por outros motivos.

Essas crianças recebem intervenção prioritariamente na área motora. O desenvolvimento neuromotor pode estar atrasado se a terapia física não se iniciar precocemente. $\mathrm{O}$ atraso motor faz a criança atuar como tendo uma idade menor e aumentar o risco de superproteção, o que é comum em famílias de deficientes.

A ausência de atividades devido à baixa estimulação visual é um problema comum entre essas crianças. Isso leva, em muitos casos, a uma auto-estimulação caracterizada, nos casos de deficiência visual, como maneirismos. Esses comportamentos, socialmente, podem ser mais incapacitantes que a própria deficiência visual.

Segundo Veitzman ${ }^{2}$, no cuidado com crianças deficientes visuais, o diagnóstico precoce e o tratamento médico adequado assumem primordial importância. A ausência total ou parcial da visão pode interferir na habilidade motora, fundamental para o processo de independência. Leal et al. ${ }^{3}$ referem que as experiências visuais são as mais numerosas e minuciosas em relação às oferecidas pelos outros sentidos.

\section{OBJ ETIVOS}

Este trabalho tem o propósito de apresentar os resultados preliminares de um estudo e comentar as características do atendimento em intervenção precoce a crianças com visão subnormal, em um hospital universitário. Assim, verificar o número de crianças com deficiência visual que chegam a um serviço de intervenção precoce em visão subnormal e que apresentam outras deficiências associadas; verificar a média de idade com que essas crianças chegam ao serviço; levantar as características específicas da população de estudo.

\section{CASUÍSTICA E MÉTODOS}

A população-alvo foi constituída por crianças na faixa etária de 0 a 3 anos, com diagnóstico de deficiência visual, moradoras em diferentes regiões do Brasil, atendidas em um serviço de estimulação em visão subnormal. Compôs-se uma amostra de tamanho 18, não probabilística, obtida segundo critério de viabilidade da coleta de dados e de facilidade do acesso ao usuário. Utilizou-se um protocolo para avaliação do desenvolvimento infantil e avaliação funcional da visão, adaptado de Knobloch \& Pasamanick ${ }^{4}$ e Barraga $^{5}$ e, avaliação oftalmológica de rotina.
Fizeram parte desse estudo, crianças atendidas num período de 3 meses durante o ano de 1998. Não pretendeu-se, portanto, generalizar os resultados.

As avaliações foram aplicadas por uma equipe multidisciplinar, composta por terapeutas ocupacionais, fonoaudiólogo e médico oftalmologista, com duração média de quarenta minutos. Os pais ou responsáveis estiveram presentes durante a realização de todos os procedimentos.

\section{RESULTADOS E DISCUSSÃO}

Verificou-se que $9(50 \%)$ dentre os 18 casos apresentaram outras deficiências, associadas à deficiência visual. Gagliardo et al. ${ }^{6}$ relatam que dentre os casos avaliados, 67,51\% apresentaram associação de deficiência visual com outras deficiências.

Sobre esse mesmo aspecto, Hyvärinen ${ }^{7}$ relata que $70 \%$ das crianças deficientes visuais possuem também outras deficiências.

Devido à dificuldade em se observar as respostas visuais nessas crianças, normalmente o possível diagnóstico oftalmológico é tardio, comprometendo ainda mais seu desenvolvimento. Geralmente é difícil de se obter ou entender as respostas dadas pelas crianças com múltipla deficiência, principalmente aquelas com quadro neurológico associado. Nesses casos, a maioria dos métodos convencionais apresenta resultados duvidosos no que se refere à estimativa da acuidade visual, devido aos comportamentos reflexos dessas crianças, que vão interferir nas respostas. Assim, Veitzman $^{2}$ relata que é urgente a participação dos oftalmologistas em equipes multidisciplinares. Da mesma forma, Hyvärinen ${ }^{8}$ sugere uma interação maior entre médicos e outros membros da equipe, pois, a partir dessa interação, os médicos adquirem mais conhecimentos sobre o funcionamento da visão em crianças com múltipla deficiência.

Verificou-se que foi de 21 meses a média de idade de chegada dessas crianças ao serviço de intervenção precoce. Observa-se que o comprometimento neuromotor pode retardar o encaminhamento dessas crianças ao oftalmologista, pois a deficiência motora é primeiramente diagnosticada. Essas crianças poderiam ser beneficiadas por uma intervenção, se a detecção e o diagnóstico fossem realizados no primeiro ano de vida. Dessa forma, obteria repercussão favorável no seu desenvolvimento como um todo, a partir da estimulação visual adequada. Sonksen et al. ${ }^{9}$ sugerem que a intervenção seja realizada no primeiro ano de vida, favorecendo um maior benefício para a eficiência visual. 
O primeiro ano de vida corresponde a um período em que o organismo está pronto para receber e utilizar os estímulos ambientais, reunindo condições satisfatórias para a aquisição e desenvolvimento das diferentes funções. Assim, esse período entre o nascimento e o primeiro ano de vida caracteriza-se como sendo um dos mais críticos no desenvolvimento da criança, o que reforça a idéia da importância de nele se diagnosticar qualquer alteração de desenvolvimento ${ }^{10}$.

A detecção e o diagnóstico precoce necessita, pois, de educação da equipe de saúde dos postos de saúde e da família.

Kara-José et al. ${ }^{11}$ sugerem que os pediatras deveriam ser conscientizados quanto à necessidade de avaliar a acuidade visual das crianças. Recomendam alertar os pediatras quanto ao papel fundamental que podem e devem desempenhar na prevenção da deficiência visual.

Em relação à variável sexo, verifica-se predominância do sexo masculino, obtendo-se uma frequiência de $72,22 \%$. Esse resultado coincide com os observados em outros estudos ${ }^{12,13}$.

\section{CONCLUSÃO}

Em crianças com múltipla deficiência o diagnóstico oftalmológico ocorre tardiamente, em geral após o diagnóstico de outras especialidades.

A avaliação oftalmológica de crianças com comprometimentos neuromotores deveria ser efetuada concomitante à avaliação neurológica e pediátrica.

A associação de quadro neurológico à deficiência visual sugere que crianças com lesão neurológica devam passar por avaliação oftalmológica, tão logo seja detectado o problema.

\section{SUMMARY}

Multiple Deficiencies and Low Vision

Children with multiple deficiencies present associated visual deficiency frequently. Generally, children with serious neurological disorders have serious motor impairment which is prioritized in programs of rehabilitation. Due to the difficulty in observing the visual answers in those children, the diagnosis of possible visual impairment is delayed. Over a period of 3 months, the authors found a frequency of $50 \%$ of children with multiple deficiency associated to the visual deficiency. It was verified that those children took 21 months on average to be seen in our service. The neuromotor impairment can delay the referral of those children to on eye specialist. Children presenting low vision can be prevented of habilitation during sensitive periods of development of the vision. This could have harmed its visual development and in consequence, the neuromotor development.

\section{KEY WORDS}

Low vision, neuromotor development, multiple deficience.

\section{Referências}

1. Zaldivar, F. \& Rubio, V. La evaluación de personas plurideficientes. Siglo Cero, 148:21-32, 1993.

2. Veitzman, S. O papel do oftalmologista numa equipe multidisciplinar para a habilitação de crianças deficientes visuais. Arq Bras Oftal, 55:215-217, 1992.

3. Leal, D.B.; Tavares, S.S.; Ventura, L.O.; Florêncio, T. Atendimento a portadores de visão subnormal: estudo retrospectivo de 317 casos. Arq Bras Oftal, 58:439-42, 1995.

4. Knobloch, H. \& Pasamanick, B. Gesell e Amatruda. Diagnóstico do desenvolvimento. Rio de Janeiro, Atheneu, 1990. 550p.

5. Barraga, N. Sensory Perceptual Development. In: School, G.T. Foundations of Education for Blind and Visually Handicapped Children and Youth. New York, American Foundation for the Blind, 1986. p.83-98.

6. Gagliardo, H.G.R.G.; Nobre, M.I.R.S.; Carvalho, K.M.M Intervenção precoce ambulatorial: orientação à família. Arq Bras Oftal, 59:352, 1996.

7. Hyvärinen, L. La visión normal y anormal en los niños. Madri, ONCE, 1988. 92p.

8. Hyvärinen, L. Considerations in evaluation and treatment of the child with low vision. American J of Occupational Therapy, 59:891-97, 1995.

9. Sonksen, P.M.; Petrie, A.; Drew, K.J. Promotion of visual development of severely visually impaired babies: evaluation of a developmentally based programme. Dev Med Child Neurol, 33:320-335, 1991.

10. Nakamura, H.Y. Investigação do comportamento auditivo em recém-nascidos e lactentes. Campinas, 1996. Dissertação de Mestrado - Universidade Estadual de Campinas.

11. Kara-José, N.; Carvalho, K.M.M.; Caldato, R.; Pereira, V.L.; Oliveira, A.M.N.D.; Fonseca Neto, J.C. Atendimento de amblíopes e prevalência na população pré-escolar, Campinas, São Paulo, Brasil. Bol Of Sanit Panamer, 97:31-37, 1984.

12. Carvalho, K.M.M. Visão subnormal: apresentação de um modelo de atendimento e caracterização das condições de diagnóstico e tratamento em um serviço universitário do Brasil. Campinas: 1993, Tese de Doutorado - Universidade Estadual de Campinas.

13. Nobre, M.I.R.S. Atendimento de estimulação em serviço de visão subnormal: características de usuários, opinião e conduta de mães. Campinas, 1996. Dissertação de Mestrado - Universidade Estadual de Campinas.

\section{Endereço para correspondência:}

CEPRE - Faculdade de Ciências Médicas - UNICAMP Av. Adolfo Lutz S/n. - Cidade Universitária Zeferini Vaz Barão Geraldo

CEP 13084-880 Campinas (SP) 